\title{
Cambios en el patrón de los usos digitales por el Covid-19. Aplica- ción del Learning Analytics a un estudio de caso entre estudiantes universitarios
}

\author{
MaCovadonga de la Iglesia Villasol \\ cvi@ccee.ucm.es \\ Universidad Complutense de Madrid, España
}

\begin{abstract}
La situación de excepcionalidad generada por la pandemia mundial COVID-19 ha obligado al uso masivo de herramientas digitales en todos los niveles educativos, evidenciando tanto desajustes en la carga docente para docentes y estudiantes en su adaptación, como la necesidad de observar con más detalle el proceso de aprendizaje que siguen los estudiantes. Este trabajo realiza una primera exploración de los cambios en los usos digitales de los estudiantes al pasar de la enseñanza presencial, con apoyo del campus virtual, a la online, según los registros o huellas que quedan al acceder a recursos virtuales. El estudio de caso se contextualiza en la asignatura de Organización Industrial del segundo cuatrimestre del curso 2020, y describe tipologías y patrones de usos diferenciados antes y después de la declaración del estado de alarma nacional, con un efecto diferenciado, siendo los estudiantes del cuartil Q1 de la distribución quienes más reactivan su actividad digital, y que ven una oportunidad de reengancharse en la docencia online, elementos que sirven de guía para una futura eventualidad.
\end{abstract}

\section{Palabras clave}

Registros digitales; brecha digital; COVID-19, analítica del aprendizaje 


\title{
Changes in the pattern of digital uses due to Covid-19. Application of Learning Analytics to a case study among university students.
}

\author{
Macovadonga de la Iglesia Villasol \\ cvi@ccee.ucm.es \\ Universidad Complutense de Madrid, España.
}

\begin{abstract}
Summary
The exceptional situation generated by the global pandemic COVID-19 has forced the massive use of digital tools at all educational levels, evidencing both mismatches in the teaching load for teachers and students in their adaptation, as well as the need to observe in more detail the learning process that students follow. This work makes a first exploration of the changes in the digital uses of students as they move from face-to-face teaching with the support of the virtual campus to the online one, according to the records or traces that remain when accessing virtual resources. The case study is contextualized in the subject Industrial Organization of the second semester of the 2020 academic year, and describes typologies and patterns of different uses before and after the declaration of the state of national alarm, with a differentiated effect, being the students in quartile Q1 of the distribution who more reactivate their digital activity, they see an opportunity to re-engage in online teaching, elements that serve as a guide for a future eventuality.
\end{abstract}

\section{Keywords:}

Digital records; digital gap; COVID-19; learning analytics. 


\section{I- Introducción}

Durante las dos últimas décadas, el sistema educativo ha incorporado numerosas herramientas digitales, reorientando la acción docente hacia el estudiante e intensificando la participación. Esto ha propiciado que los docentes, sin dejar de ser expertos en contenidos, desarrollen altos grados de competencias digitales, yendo desde el desarrollo y validación de materiales y metodologías docentes innovadoras en entornos virtuales de aprendizaje, a la extracción y análisis de los registros digitales que quedan cuando los usuarios acceden a los mismos (Berners-Lee, Hendler y Lassila, 2001). La captura, gestión y análisis de dichos registros en las herramientas digitales aportan matrices de información de gran tamaño que requiere en muchos casos software de procesamiento específico para sistematizar la información significativa. (Mazza y Milani, 2004; Mazza y Botturi, 2007; Romero et al., 2008).

La variedad de contextos, tipos de cursos, metodologías, o criterios de evaluación, hace de las Learning Analytics (LA) una herramienta útil para las instituciones para abordar algunos de los desafíos actuales en los procesos de enseñanza, como la deserción temprana de estudiante; generalmente las tasas de abandono o deserción son mayores en el aprendizaje digital respecto al presencial (Yukselturk, Ozekes y Turel, 2014), o el bajo rendimiento, vinculados a las metodologías aplicadas, el perfil diferenciado de los estudiantes, la gestión autónoma del tiempo, el compromiso con el programa formativo y asumir responsabilidades (OCDE, 2019).

La amplia investigación empírica de las LA en la última década, aporta diseños simples aplicables a cualquier curso, accesibles y adaptables para todos los docentes, sin necesidad de un software específico, basados en descripciones de registros genéricos que ofrecen las plataformas de campus virtual. Estos diseños son reutilizables de unos cursos a otros y reescalables a otros niveles de aprendizaje. Así, el uso masivo de las herramientas online generado en el periodo actual de la pandemia COVID-19, está abriendo desafíos específicos de evaluación en nuevos escenarios, incrementando exponencialmente los registros que dejan los estudiantes. El uso de la tecnología en los entornos virtuales de aprendizaje visualiza el grado de compromiso de los estudiantes con el programa, según la intensidad de las interacciones con las diferentes actividades en las aulas virtuales (Li, Baker y Warschauer, 2020).

La docencia online o presencial con apoyo virtual, genera un gran volumen de datos, condicionado al modelo de enseñanza y el cronograma (actividades docentes y de evaluación) (Baker e Inventado, 2014). El alcance y objetivos de las investigaciones empíricas difieren según: 1) las fuentes de datos utilizados en los análisis (demográficos, encuestas, evaluaciones), 2) el nivel educativo de la cursos, 3) el objetivo de los modelos descriptivos, predictivos, de intervención, 4) el alcance del análisis (programa formativo, curso o disciplina), 5) la modalidad del curso (presencial, semipresencial, a distancia) y 6) las técnicas utilizadas en la clasificación y análisis (Chick y otros, 2020, y García-Peñalvo y otros, 2020).

Este trabajo analiza los datos de registros del uso del campus virtual Moodle, usos de los usuarios, tipología de recursos, patrones y frecuencias, durante el segundo semestre del curso 2019-20, aplicando técnicas descriptivas que transforman datos de registros electrónicos en información y conocimiento, revelando correlaciones y patrones ocultos (Liz-Domínguez y otros, 2019). El estudio de caso consiste en la captura, procesamiento, comprensión y modelado de los datos, para generar feed-back significativo, quedando fuera del mismo un estudio diagnóstico o prescriptivo. 


\section{Contextualización teórica}

El cambio transitado por cualquier sistema educativo está afectando a la forma en que se administra y gestiona la infraestructura tecnológica que genera bases de datos a gran escala, disponibles para profesores y administración, y de forma más limitada para los propios estudiantes. La presencia del LA en el entorno educativo es ya una realidad, y puede formar parte de algunas soluciones, habiendo sido definido1 como la recopilación, medición, análisis y presentación de datos sobre los estudiantes y sus contextos, para comprender y optimizar el aprendizaje y los entornos en que se produce (Long, Siemens, Conole y Gašević, 2011). Dada la extensísima literatura, teórica y empírica del LA, nos referiremos únicamente a algunos aspectos vinculados directamente con el estudio de caso que se presenta, dado que hacer una revisión exhaustiva de la misma excede los límites y objetivos del mismo.

Este fenómeno, el uso intensivo del LA, aúna dos aspectos claves como son i) la disponibilidad de matrices de datos de gran tamaño generadas por el uso masivo de herramientas digitales, y ii) la generación de nuevas áreas de investigación con una masiva producción científica teórica y empírica, bajo enfoques distintos (por ejemplo el Analytics o Educational Data Mining, EDM, o el Learning Analytics, LA), más de grado, objetivo o intención2 que de metodología y alcance, (Campbell, DeBlois y Oblinger, 2007; Romero et al., 2008, Long y Siemens, 2011). En concreto, el LA aborda aspectos históricamente desconocidos en el proceso de aprendizaje-aprendizaje principalmente con análisis descriptivos y predictivos si se aplica en etapas tempranas, si bien aspira a extraer informes diagnósticos o prescriptivos en un futuro para, por ejemplo atender a necesidades precisas de aprendizaje de estudiantes (Campbell et al., 2007), identificar la relación entre el rendimiento académico y las interacciones digitales, (Ramos y Yudko, 2008; Iglesia 2020), o entre la participación, la interacción digital y las tasas de abandono y/o deserción (Cocea y Weibelzahl, 2007; Xing, Guo, Petrakovic y Goggins, 2015). Estos temas se abordan con apoyo de algoritmos de naturaleza distinta según el carácter descriptivo o predictivo, que van desde la optimización de hiperparámetros basados en el recuento, o la búsqueda de la cuadrícula (grid search), con aplicaciones principalmente en el entorno universitario, siendo una buena guía Liz-Domínguez y otros (2019) y Herodotou, Rienties, Verdin y Boroowa (2019).

Los beneficios del LA para usuarios e instituciones (Picciano, 2012 y 2014), son 1) el monitoreo del desempeño y progreso de los estudiantes (frecuencia de acceso, tiempo, número y naturaleza de las interacciones, contenidos); 2) aportar datos detallados sobre la instrucción, asesoramiento, vincular actividades educativas con datos de otra naturaleza, institucional, de rendimiento, etc., (preparación, género, edad, calificaciones de acceso, notas expediente); 3) proporcionar análisis longitudinal con carácter predictivo del comportamiento futuro, estableciendo sistemas de alerta y estrategias tempranas de intervención. Extraer información sólida y significativa requiere atender a: 1) que los datos sean precisos, oportunos, fiables, vinculados a que existan investigadores expertos conocedores de árboles de decisión, estrategias de mapeo o algoritmos de modelos predictivos; 2) gestionar matrices de datos de gran tamaño con un software complejo sin difuminar los límites de lo racional en la toma de decisiones, evitando generar ruido estadístico o predicciones inútiles o perjudiciales; y 3) tener en cuenta la privacidad y el derecho que asiste a los usuarios o estudiantes (Sanger y Perlroth, 2014).

1 Definida en la 1st International Conference on Learning Analytics and Knowledge (LAK 2011) de la Society for Learning Analytics Research (SoLAR).

2 Referidas la primera al estudio del comportamiento e interacciones de los estudiantes en los contextos digitales, y la segunda a la medición, recopilación, análisis e informe de los alumnos en el aprendizaje. 
Este campo de estudio tiene un enfoque claramente multidisciplinar, fusionado con diferentes áreas como la estadística, la ciencia computacional, la pedagogía, el aprendizaje automático o la inteligencia artificial (Banihashem y otros, 2018), siendo el nexo que une todas ellas el concepto de aprendizaje, optimizarlo y mejorarlo, a partir del conocimiento que aporta el LA (Long y Siemens, 2011; Gašević, Dawson y Siemens, 2016). Pero también puede ser objeto de una brecha entre el enfoque teórico y el ejercicio práctico (Stewart, 2017), por lo que Siemens (2013) y Knight, Shum y Littleton (2014) mencionan la amenaza del uso extensivo y determinista de la tecnología en el LA. La introducción del LA en los entornos educativos debe ir acompañado del diseño de un modelo teórico que oriente sobre qué información extraer y gestionar, analizar y sintetizar, el uso de la misma, descriptivo, predicción o intervención, respondiendo a la pregunta de cuál es el papel de la teoría del aprendizaje en los diversos contextos de aplicación del LA. En concreto se fijan tres roles: el papel de soporte o apoyo, el papel rector y el papel del sentido (underpinning, guiding, sense-making), en tres pasos: 1) dónde es mejor aplicar el uso de los modelos y análisis, 2) el tipo de datos que se deben recopilar para procesar los informes, y 3) interpretar apropiadamente los hallazgos e las intervenciones educativas apropiadas (Banihashem y otros, 2019).

Caben diversos niveles de análisis empírico del LA (Buckingham y Ferguson, 2012), según desagregación: análisis macro (estado, región o provincia), análisis meso o institucional (centros educativos, titulaciones) y nivel micro (curso, grupo, aula), siendo éste el que aporta mayor desagregación, cuyo uso temprano y periodificado puede llegar a describir los hábitos de aprendizaje de los estudiante e identificar a aquellos con insuficiente actividad digital o con mayor riesgo de abandono del curso. Durall y Gros (2014) y Gros (2016) consideran que el LA genera un feedback formativo para los distintos agentes sobre el comportamiento, compromiso y aprendizaje de los estudiantes que explora el aprendizaje con objetivos, enfoques y metodologías diversos (Siemens, 2013). Gašević, Dawson, Rogers y Gasevic (2016) afirman que los modelos de LA más generales tienen menor éxito contrastado en las predicciones que los modelos aplicables a un curso específico, no ajustándose de igual forma a todos los contextos de aprendizaje, siendo más plausible su aplicación en los estudios de caso.

Uno de los futuros debates, hoy aún incipiente, es la importancia de cumplir con los requerimientos de privacidad de los usos de los estudiantes, (Campbell, 2007), que se extenderá a aspectos como el contexto en el que se realizarán los análisis, los fines, cumplir los criterios de anonimato, o regular el intercambio de datos entre plataformas, redes de investigadores u organizaciones. Drachsler y Greller (2016) avanzan en esta línea al detallar diversos ítems de verificación, lista DELICATE3, garantizando que el registro de los datos de las interacciones de los estudiantes no dañe su privacidad, requiriendo protocolos de protección, éticamente admisibles y estructuras de consentimiento explícito. Mientras que el big data, y por tanto el LA, es éticamente neutral, su uso sin embargo puede no serlo, por lo cual el investigador debe alinear el objetivo de la investigación con los derechos y valores de los usuarios (Davis, 2012), en la transparencia, seguridad y responsabilidad de las acciones cotidianas como son los cursos online.

3 Determination (propósito); Explain (alcance y uso); Legitimate (marcos legales); Involve (acceso a las partes interesadas, garantía, uso); Consent (consentimiento explícito); Anonymise (anonimato), Technical (supervisión y External (cumplimiento de seguridad). 


\section{Objetivo y método del estudio de caso}

\section{a. Objetivo}

El estudio de caso tiene como objetivo identificar los cambios en los usos digitales de los estudiantes universitarios como consecuencia de la adaptación de la docencia presencial con apoyo del campus virtual, a una docencia exclusivamente online consecuencia de la declaración del estado de alarma por la pandemia covid-19.

Es un análisis a nivel micro (Buckingham y Ferguson, 2012), que identifica los cambios en los hábitos y patrones de usos digitales de los estudiantes, y ayuda a conocer mejor la propia acción docente, la dependencia de dichos usos digitales al cronograma de la programación didáctica y de evaluación, así como la interacción los estudiantes con las herramientas digitales, sus actitudes y el compromiso con el programa formativo, para disponer de un feedback en el proceso futuro de diseño e-learning de la materia.

\section{b. Metodología}

\section{Diseño y características de los participantes}

El análisis es cuantitativo de tipo descriptivo, a partir de la gestión de la información extraída de los registros de los accesos y usos que los estudiantes hacen en las plataformas digitales enlazadas en el campus virtual (Moodle). La propia limitación de los microdatos que se graban en los registros del Moodle imposibilita un estudio de carácter predictivo o experimental, si bien aporta información relevante para una evaluación de los cambios en la actitud y actividad digital de los estudiantes.

Se particulariza para la asignatura de Organización industrial (OI) de cuarto curso del itinerario de Análisis Económico ( $\mathrm{AE}$ ), del Grado en Economía (GECO), de la Universidad Complutense de Madrid, impartida en el segundo cuatrimestre del curso 2019-20. La población objeto de estudio es el total de estudiantes, sin realizar muestreo probabilístico, formada por 85 estudiantes matriculados, excluidos aquellos que en las dos primeras semanas anulan su matrícula o cursan el programa de Erasmus-Out en otras Universidades.

La población del estudio se caracteriza porque: i) el 62,35\% del total se matriculan con un DNI de nacionalidad española, y el resto, 37,65\% con NIE4 de nacionalidad extranjera, siendo el 22,35\% de nacionalidad China; ii) El 63,52\% del total son varones, porcentaje muy próximo al de la titulación $64,7 \%$, que difiere respecto del porcentaje del centro (58,6\% de varones), y iii) el $64,71 \%$ del total cursan la asignatura en primera convocatoria, el $12,94 \%$ en segunda y el $21,17 \%$ en tercera.

\section{Instrumentos y procedimiento}

Para afrontar el objetivo propuesto, se utilizan diversas fuentes de información primaria (Sampson, 2017): una matriz de gran tamaño con los registros de acceso y usos digitales en las plataformas del campus virtual y bases que caracterizan a los individuos, aportan su evaluación y participación en el curso, anidadas por el documento de matrícula. En este sentido, Lodge y Corrin (2017) señalan lo importante de disponer de información amplia, sistemática e integral sobre diferentes aspectos del proceso de aprendizaje, desde distintas bases de datos, perfectamente enlazadas. Concretamente, las fuentes son:

4 NIE es el número de identidad de extranjero, bien por residencia temporal o por estudios. 
- Información Institucional, según datos de matrícula de los estudiantes: identificación, convocatoria, estudiantes Erasmus, años en la Universidad, etc.

- Información de Registros en el campus virtual Moodle.

- Información sobre rendimiento y evaluación en el curso.

- Los registros digitales se han extraído y gestionado según Iglesia (2019), tanto en la descripción como el análisis estadístico, en correspondencia con Kay, Kom y Oppenheim5 (2013) y Drachsler y Greller (2016), respetando ciertos posicionamientos éticos (Pardo y Siemens, 2014 y Slade y Prinsloo, 2013)6.

- La recogida de datos se realiza durante el curso académico 2019/2020, en dos fases: 1) la información institucional, invariante a lo largo del período lectivo, se extrae al comienzo del mismo (febrero de 2020); 2) la información de los registros digitales se extrae durante y al finalizar el periodo lectivo (abril y finales de mayo de 2020): Se han utilizado herramientas básicas del Excel y de SPSS, respetando escrupulosamente la identidad de los usuarios7.

\section{Resultados}

Se presenta un análisis de carácter exploratorio y descriptivo a partir de estadísticos básicos para identificar algunos patrones en los registros de acceso y usos digitales, en las diversas fases del curso, previo al inicio de la actividad docente, fase presencial y fase online, de 8 semanas cada una de éstas últimas, fechadas antes y después de la declaración del estado de alarma (14 de marzo 2020).

\section{a. Exploración de los registros digitales y brecha digital.}

Se registraron 59.152 accesos digitales en la plataforma Moodle, desde el 2 de septiembre del 2019, fecha de activación virtual de la asignatura de referencia. A este tipo de registros se refiere Siemens (2013) como las huellas que dejan los usuarios en los senderos digitales, y del total señalado, se excluyen 136 al ser accesos del "Administrador del Sistema" y 194 de "Usuarios servicios Web", que representan conjuntamente el $0,56 \%$ del total, siendo 58.822 el número neto de accesos válidos.

Particularizando, el 6,63\% de los registros son del profesor, explicado por la intensa actividad de adaptación metodológica de la docencia presencial con apoyo virtual a la exclusivamente virtual, consecuencia de la pandemia sobrevenida a la COVID-19. Del total de registros cabe distinguir tres periodos temporales claros, tabla 1, con dispar frecuencia en la actividad, total y según el rol de profesor o alumno: El 3,84\% de los accesos (2260) se realizan previo al periodo lectivo de la asignatura del segundo cuatrimestre (del 2 de septiembre de 2019 al 19 de enero de 2020), perteneci-

5 Identifican rangos diversos de datos: i) Actividad registrada en cursos virtuales, ii) Interacciones de sistemas de búsqueda de información, iii) Mecanismos de retroalimentación, y iv) sistemas externos de identificación compartidos.

6 Solicitar el consentimiento, informar sobre el propósito de recopilar datos y garantizar un uso exclusivamente para el propósito notificado en los términos legales.

7 Se informa a los estudiantes sobre la extracción de los registros digitales, pudiendo solicitar ser excluidos, sin que el consentimiento suponga compromiso académico, obligación o dedicación adicional para el estudiante, ni interferencia en las calificaciones. 
endo el 0,36\% al profesor, mostrando una intensa actividad de los estudiantes por acceder a la información del campus virtual. El 16,30\% de los registros se realizan en las 8 primeras semanas de periodo lectivo presencial, la actividad se intensifica hasta el $79,86 \%$ en las 8 semanas lectivas online en periodo de alarma nacional, casi 5 veces más (4,90 veces más), que muestra la fuerte presión que las circunstancias sanitarias ha supuesto sobre el campus virtual. Mientras que la actividad virtual del docente se ha multiplicado por más de 10 veces $(10,63)$ al actualizar y aportar nuevos recursos, impartir docencia, tutorías, programar y evaluar pruebas online, la de los estudiantes solo por 4,70 veces.

\begin{tabular}{lccc}
\hline Periodo & Total & Profesor & Alumnos \\
\hline PREVIO al Inicio actividad lectiva & $3,84 \%$ & $0,36 \%$ & $3,48 \%$ \\
PRESENCIAL: 8 semanas (20 enero-14 marzo, & $16,30 \%$ & $0,54 \%$ & $15,76 \%$ \\
$2020)$ & $(1198,5)$ & $(39,375)$ & $(421,75)$ \\
ONLINE: 8 semanas (16 marzo-29 mayo, 2020) & $79,86 \%$ & $5,74 \%$ & $74,12 \%$ \\
& $(5871,62)$ & $(5449,87)$ & $(1159.13)$ \\
\hline Total registros (N=58822) & $100 \%$ & $6,63 \%$ & $93,37 \%$ \\
\hline
\end{tabular}

Tabla 18. Porcentaje y media semanal registros según fases temporales y rol.

Esta primera observación apunta a diversos patrones de uso digital, antes y después del estado de alarma, pasando el campus virtual de ser un apoyo a la docencia presencial a ser un necesario sustituto de la misma, tanto en la frecuencia, como en las pautas y elementos a los que se accede (gráfico 1).

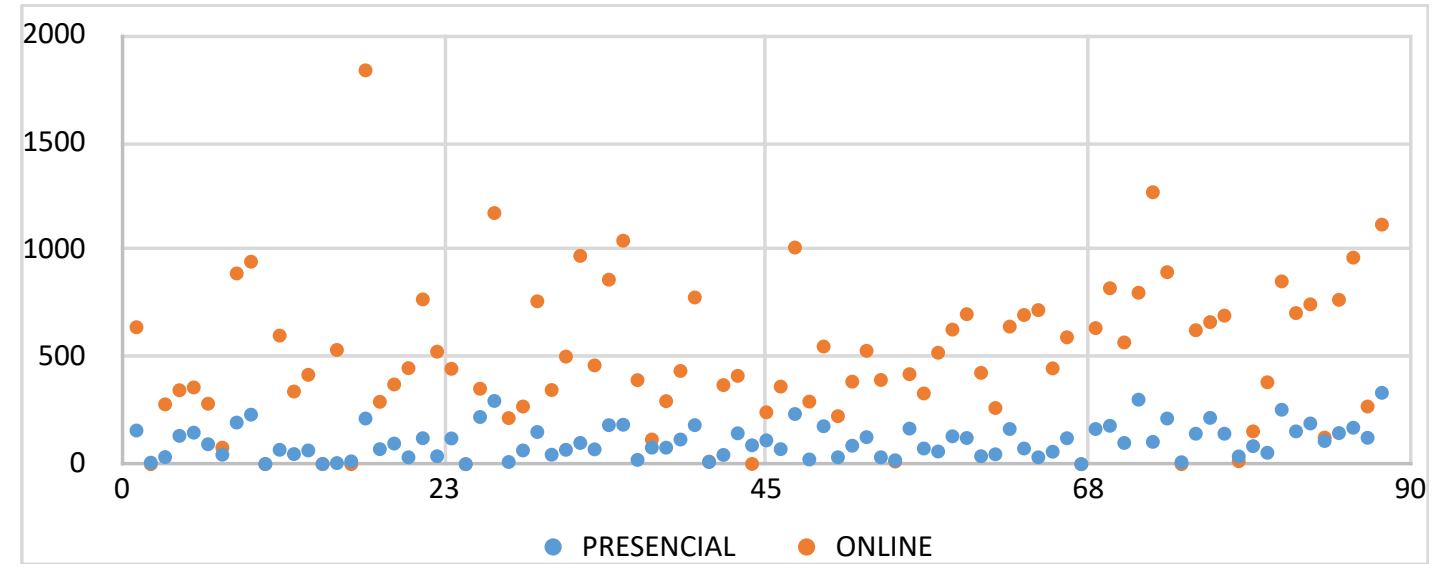

Gráfico 1. Frecuencia de registros por alumno, según fases, presencial u online.

Los perfiles asimétricos en los registros de los estudiantes, ordenados de mayor a menor frecuencia, con diferencial entre la fase presencial y online, gráfico 2, ajustados por una la línea de tendencia lineal con claras diferencias tanto en la ordenada como en su pendiente, que muestra la mayor desigualdad en los registros en la fase online $(-12,675$ frente a $-2,899$ en la fase presencial). Complementariamente, el gráfico 3 aporta los gráficos de dispersión para ambas fases, y confirma las diferencias de nivel en la contribución al total de registros, así como la línea de ajuste con pendientes de 0,818 y 0,158 respectivamente en la fase online y presencial.

8 Todas las tablas y gráficos son de elaboración propia 


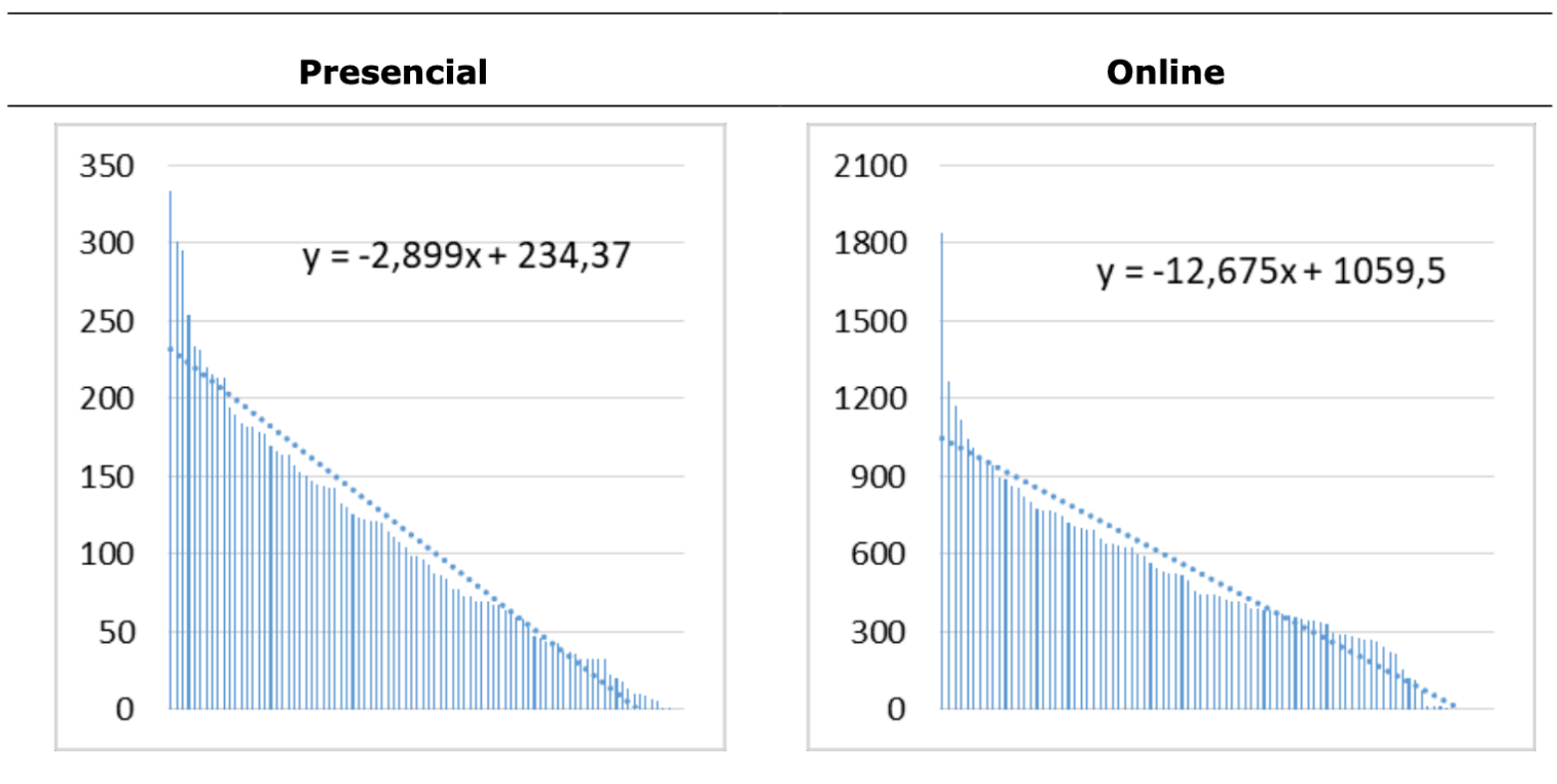

Gráfico 2. Registros ordenados de mayor a menor, según fase.

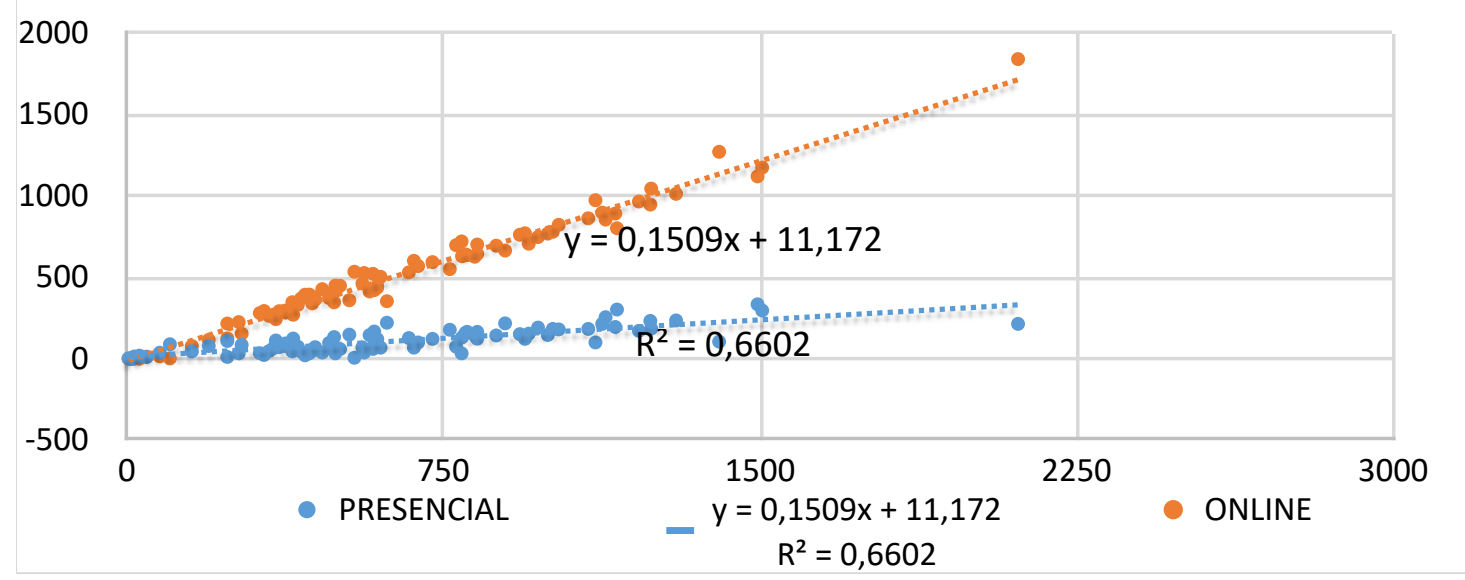

Gráfico 3. Dispersión de los registros, según fase.

La media de los registros es 495,44 en la fase online y 105,36 en la presencial (4,7 veces más), siendo la diferencia con el total los registros del período previo al inicio de la docencia (tabla 2). El cuartil Q1 muestra que el $25 \%$ de los alumnos es menor o igual que 43,75 en fase presencial y 281,25 en online (6,42 veces más), mientras que en el cuartil Q2 esa mayor intensidad se multiplica por 4,65 veces, y el Q3 muestra que el $75 \%$ de los datos es menor o igual a 154 en docencia presencial y 701,25 en online (4,55 veces más): así, la mayor desigualdad entre fases se observa en el cuartil Q1, siendo éstos los que más reactivan su actividad digital. El coeficiente de asimetría respecto del promedio de registros refuerza esta información: 0,714 en fase presencial y de 0,831 en la online. 


\begin{tabular}{lcccc}
\hline & Total & Presencial & Online & \% Online del Total \\
\hline Máximo & 2111 & 333 & 1838 & 87,06 \\
Mínimo & 4 & 0 & 0 & 0 \\
Media & 624,09 & 105,36 & 495,44 & 70,37 \\
Cuartil Q1 & 359 & 43,75 & 281,25 & 71,67 \\
Cuartil Q2 (Mediana) & 560,01 & 94,50 & 440,0 & 78,24 \\
Cuartil Q3 & 880,25 & 154,0 & 701,25 & 84,27 \\
\hline
\end{tabular}

Tabla 2. Descriptivos básicos de los registros de estudiantes según fases temporales.

Las curvas de concentración (gráfico 4) calculada a partir de los porcentajes de participación de cada estudiante por fase, acumulados mayor a menor, se acoplan bastante bien sin que exista excesiva desigualdad entre ellas, excepto en la zona central de la misma, si bien es menor la concentración en la fase online (menor concavidad). Esta información se refuerza con el índice CR10 de concentración (la suma de la participación en el total de los 10 alumnos con mayor frecuencia de registros), con valores del $27,07 \%$ y $25,75 \%$ en la fase presencial y online, respectivamente. Este CR10 se complementada con el índice Herfindahl (IH)9 (sumatorio de las proporciones al cuadrado), con valores respectivamente de 0,01727 y 0,01665 en las fases presencial y online, que determinan un número equivalente respectivamente de 57,88 y 60,03 , siendo éste el número "hipotético" de alumnos con igual frecuencia de registros que determinarían el igual índice IH calculado (tabla 3).

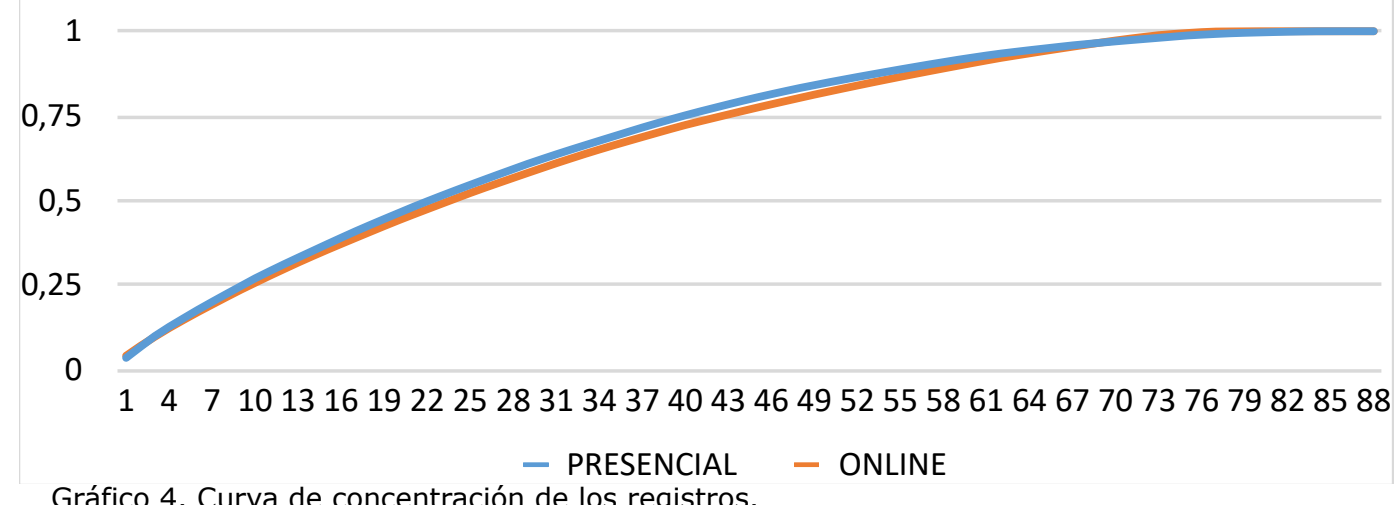

Gráfico 4. Curva de concentración de los registros.

9 Los índices CRi y Herfindahl-Hirschman (IH) son conceptos habituales en el estudio empírico de la asignatura de referencia OI y que cabe aplicar en este estudio. IH toma valores entre $1 / \mathrm{N}$ y 1 , siendo $\mathrm{N}$ el número de individuos. 


\begin{tabular}{lccc}
\hline & Total & Presencial & Online \\
\hline Indice de concentración $\mathrm{CR}_{10}$ & $25,18 \%$ & $27,07 \%$ & $25,75 \%$ \\
Indice Herfindahl IH & 0,01625 & 0,01727 & 0,01665 \\
Número Equivalente, NE=1/IH & 61,55 & 57,88 & 60,03 \\
\hline
\end{tabular}

Tabla 3. Indices de Concentración, Herfindhal y número equivalente.

A continuación, el análisis se desagrega recogiendo el análisis particularizado de los 10 estudiantes más y menos activos digitalmente, exclusivamente en las fases objeto de estudio, presencial y online (tabla 4).

\begin{tabular}{|c|c|c|c|c|c|c|c|c|c|}
\hline & $\begin{array}{l}\% \\
\text { to- } \\
\text { tal }\end{array}$ & $\begin{array}{c}\text { Media } \\
\text { semanal }\end{array}$ & $\begin{array}{c}\text { DIF(*) } \\
\text { respec- } \\
\text { to me- } \\
\text { dia } \%\end{array}$ & $\begin{array}{c}\text { Brecha } \\
\text { digital, } \\
\%\end{array}$ & & $\begin{array}{c}\% \\
\text { total }\end{array}$ & $\begin{array}{l}\text { Media } \\
\text { semanal }\end{array}$ & $\begin{array}{c}\text { DIF } \\
\text { respec- } \\
\text { to me- } \\
\text { dia } \%\end{array}$ & $\begin{array}{c}\text { Brecha } \\
\text { digital, } \\
\%\end{array}$ \\
\hline $\begin{array}{l}10 \text { más } \\
\text { activos }\end{array}$ & & Fase & resencial & & $\begin{array}{c}10 \\
\text { más } \\
\text { acti- } \\
\text { vos }\end{array}$ & \multicolumn{4}{|c|}{ Fase online } \\
\hline A88 & $\begin{array}{c}3,5 \\
9\end{array}$ & 41,62 & 68,36 & 216,05 & A17 & 4,22 & 229,75 & 73,04 & 270,98 \\
\hline A71 & $\begin{array}{c}3,2 \\
5\end{array}$ & 37,62 & 65,00 & 185,68 & A72 & 2,91 & 158,62 & 60,96 & 156,13 \\
\hline A26 & $\begin{array}{c}3,1 \\
8\end{array}$ & 36,87 & 64,28 & 179,98 & A26 & 2,69 & 146,5 & 57,73 & 136,56 \\
\hline A81 & $\begin{array}{c}2,7 \\
4\end{array}$ & 31,75 & 58,52 & 141,07 & A88 & 2,56 & 139,75 & 55,68 & 125,66 \\
\hline A47 & $\begin{array}{c}2,5 \\
2\end{array}$ & 29,25 & 54,97 & 122,09 & A35 & 2,39 & 130,37 & 52,50 & 110,52 \\
\hline A9 & $\begin{array}{c}2,4 \\
9\end{array}$ & 28,875 & 54,39 & 119,24 & A47 & 2,32 & 126,37 & 50,99 & 104,06 \\
\hline A25 & $\begin{array}{c}2,3 \\
7\end{array}$ & 27,50 & 52,11 & 108,80 & A32 & 2,23 & 121,50 & 49,03 & 96,19 \\
\hline A76 & $\begin{array}{c}2,3 \\
3\end{array}$ & 27,00 & 51,22 & 105,00 & A86 & 2,21 & 120,50 & 48,61 & 94,57 \\
\hline A17 & $\begin{array}{c}2,3 \\
0\end{array}$ & 26,62 & 50,53 & 102,16 & A9 & 2,17 & 118,12 & 47,57 & 90,74 \\
\hline A73 & $\begin{array}{c}2,3 \\
0\end{array}$ & 26,62 & 50,53 & 102,16 & A73 & 2,06 & 112,0 & 44,71 & 80,85 \\
\hline Media & $\begin{array}{c}2,7 \\
1\end{array}$ & 31,37 & 56,99 & 138,22 & Media & 2,58 & 140,35 & 54,08 & 126,63 \\
\hline
\end{tabular}




\begin{tabular}{|c|c|c|c|c|c|c|c|c|c|}
\hline $\begin{array}{l}10(* *) \\
\text { menos } \\
\text { activos }\end{array}$ & & Fase & resencial & & $\begin{array}{c}10 \\
\text { menos } \\
\text { activo }\end{array}$ & & & e online & \\
\hline A36 & $\begin{array}{c}0,2 \\
2\end{array}$ & 2,5 & $-426,82$ & $-81,02$ & A50 & 0,51 & 28 & $-121,18$ & $-54,79$ \\
\hline A54 & $\begin{array}{c}0,1 \\
9\end{array}$ & 2,25 & $-485,35$ & $-82,92$ & A27 & 0,49 & 26,87 & $-130,44$ & $-56,60$ \\
\hline A16 & $\begin{array}{c}0,1 \\
4\end{array}$ & 1,62 & $-710,49$ & $-87,66$ & A79 & 0,35 & 19,12 & $-223,82$ & $-69,12$ \\
\hline A27 & $\begin{array}{c}0,1 \\
1\end{array}$ & 1,25 & $-953,64$ & $-90,51$ & A84 & 0,28 & 15,5 & $-299,55$ & $-74,97$ \\
\hline A41 & $\begin{array}{c}0,1 \\
1\end{array}$ & 1,25 & $-953,64$ & $-90,51$ & A37 & 0,26 & 14,37 & $-330,82$ & $-76,79$ \\
\hline A74 & $\begin{array}{c}0,1 \\
0\end{array}$ & 1,12 & $-1070,71$ & $-91,46$ & A7 & 0,18 & 9,62 & $-543,43$ & $-84,46$ \\
\hline A2 & $\begin{array}{c}0,0 \\
8\end{array}$ & 0,87 & $-1405,19$ & $-93,36$ & A78 & 0,03 & 1,75 & $-3438,88$ & $-97,17$ \\
\hline A15 & $\begin{array}{c}0,0 \\
5\end{array}$ & 0,62 & $-2007,27$ & $-95,25$ & A41 & 0,03 & 1,50 & $-4028,69$ & $-97,58$ \\
\hline A10 & $\begin{array}{c}0,0 \\
1\end{array}$ & 0,12 & $\begin{array}{c}-10436,3 \\
6\end{array}$ & $-99,05$ & A54 & 0,03 & 1,50 & $-4028,69$ & $-97,58$ \\
\hline A14 & $\begin{array}{c}0,0 \\
1\end{array}$ & 0,12 & $\begin{array}{c}-10436,3 \\
6\end{array}$ & $-99,05$ & A44 & 0,00 & 0,12 & $-49444,31$ & $-99,80$ \\
\hline Media & 01,01 & 1,17 & $-2888,58$ & $-91,08$ & Media & 0,22 & 11,84 & $-6258,98$ & $-80,89$ \\
\hline
\end{tabular}

Tabla 4. Estadísticos registros por fases y brecha de uso semanal.

Notas: $(*)$ DIF indica diferencial, $(* *)$ Excluidos los abandonos anteriores a la fase lectiva. Sombreados los alumnos que se mantienen en el ranking de los 10 más o menos activos entre fases.

Los alumnos más y menos activos no son los mismos en las fases presencial y final, lo cual evidencia un cambio de actitud y compromiso con el programa docente online: así, de los 10 alumnos más activos en la fase presencial, solo permanecen 6 en la online, mientras que de los 10 más inactivos, solo se mantienen 3 en esta clasificación, hecho reforzado por los coeficientes de asimetría señalados anteriormente (tabla 4).

Destacar que en la fase presencial los 10 estudiantes más activos ( $27,07 \%$ del total de registros), tienen una media semanal de 31,37 registros (entre 41,62 y 26,62 ), mientras que los más inactivos aportan únicamente el $1,01 \%$ del total, con media semanal de 1,17 accesos. Paralelamente, en la fase online, los 10 estudiantes más activos computan el 25,75\% de los registros totales, con media semanal de 140,35 (entre 229,75 y 112,0 ), mientras que los 10 menos activos aportan conjuntamente el 2,17\%, con media de 0,22 registros. La frecuencia de los accesos y la actividad registrada en el campus virtual difiere tanto por días como por semanas, acompasada con el cronograma de la programación docente. En cuanto a los diferenciales respecto de la media, para los 10 alumnos más activos en la fase presencial es 56,99 y 54,08 en la online. Esta información se com- 
plementa con el indicador de brecha digital10 (Iglesia, 2019) que para los 10 más activos pasa de 138,22 en la fase presencial a 126,63 en la online, y entre los 10 estudiantes menos activos pasa de $-91,08$ a $-80,89$. Este concepto difiere del enfoque teórico de Fernández Enguita (2020) y García-Peñalvo (2020)11, quienes señalan tres tipos de brechas en el entorno digital: de acceso (falta de medios o deficiente despliegue), de uso (déficit de dispositivos) y competencial (carencias en competencias digitales).

Un ejemplo de cómo los estudiantes, según su uso y/o rendimiento, pueden ser identificados por el LA está en Pardo (2018) para quien la tecnología está al servicio de la observación del proceso de aprendizaje, y Tempelaar (2020) quien clasifica a los estudiantes en cuatro cluster (deep learners, step learners, maladaptatives, adaptative) según los enfoques de aprendizaje.

Las diferencias en la brecha digital por estudiante se muestran en el gráfico 5 de dispersión con una pendiente de ajuste lineal positiva de 1,0417, con un coeficiente de correlación de Pearson de 0,9858 . Y no sólo se observan en los extremos de la clasificación de estudiantes, sino en todas las categorías (tabla 4 y 5 ). En las tipologías proactivos, sobreactivos y subactivos, la brecha digital es más moderada en la fase online $(122,354$ frente a 128,$890 ; 46,334$ frente a 51,855 y $-42,360$ frente a -49,522) siendo al contrario entre los activos (en media $-5,636$ frente a 2,608 ) e inactivos $(-96,413$ frente a $-91,531)$.

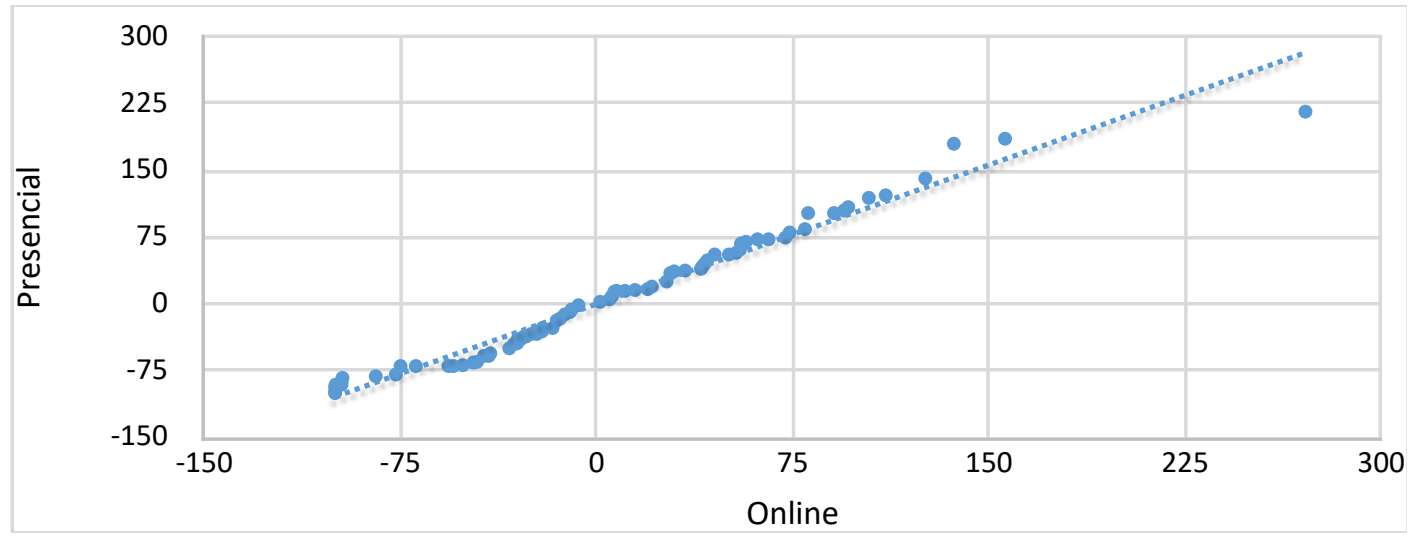

Gráfico 5. Dispersión de la Brecha digital total de estudiantes.

10 Se calcula como el diferencial respecto al valor medio, expresado porcentualmente.

11 No se tiene constancia de que los estudiantes sufran alguna desigualdad a las que se refiere. Tampoco es el caso de la brecha por resistencia al cambio del profesorado ni falta de estrategia institucional y del docente ante la situación sobrevenida por el estado de alarma covid-19. 


\begin{tabular}{lcc}
\hline & $\begin{array}{c}\text { Presencial } \\
\text { Brecha Media } \\
\text { (No Alumnos) }\end{array}$ & $\begin{array}{c}\text { Online } \\
\text { Brecha Media } \\
\text { (No Alumnos) }\end{array}$ \\
\hline $\begin{array}{l}\text { Proactivos: } \\
\text { Brecha positiva superior al } 75 \%\end{array}$ & $128,890(12)$ & $122,354(11)$ \\
$\begin{array}{l}\text { Sobreactivos: } \\
\text { Brecha positiva entre } 75 \text { y } 25 \%\end{array}$ & $51,855(18)$ & $46,334(20)$ \\
$\begin{array}{l}\text { Activos: } \\
\text { Brecha entre }+25 \text { y -25 \% }\end{array}$ & $2,608(18)$ & $-5,636(23)$ \\
$\begin{array}{l}\text { Subactivos, } \\
\text { Brecha negativa entre } 25 \text { y } 75 \%\end{array}$ & $-49,522(27)$ & $-42,360(21)$ \\
$\begin{array}{l}\text { Inactivos: } \\
\text { Brecha negativa superior al } 75 \%\end{array}$ & $-91,531(13)$ & $-96,413(13)$ \\
\hline
\end{tabular}

Tabla 5. Clasificación de estudiantes según la brecha digital.

\section{b. Tipología y temporalidad en el uso digital}

La tipología de los accesos realizados a las herramientas digitales se muestra en la tabla 6 adjunta, en la cual algunos ítems se han recodificado para facilitar su análisis:

\begin{tabular}{lccccc}
\hline & & $\begin{array}{c}\text { Presencial } \\
\text { \%Total }\end{array}$ & $\begin{array}{c}\text { Online } \\
\text { \%ototal }\end{array}$ & $\begin{array}{c}\text { Total } \\
\text { Tasa Va- } \\
\text { riación }\end{array}$ \\
\hline Archivos enviados & R1 & 1,72 & 1,64 & 1,654 & $-4,65$ \\
Chat, Foro & R2 & 8,82 & 6,10 & 6,57 & $-30,84$ \\
Collaborate & R3 & 2,78 & 5,13 & 4,73 & 84,53 \\
Cuestionario & R4 & 9,52 & 12,31 & 11,84 & 29,31 \\
Informe general & R5 & 0,04 & 0,00 & 0,01 & $-100,00$ \\
Papelera de reciclaje & R6 & 0,22 & 0,00 & 0,04 & $-100,00$ \\
Recursos, glosario, Url & R7 & 23,35 & 21,33 & 21,67 & $-8,65$ \\
Sistema & R8 & 40,75 & 44,86 & 44,16 & 10,09 \\
Tarea, Detector plagio Unicheck & R9 & 11,16 & 8,11 & 8,62 & $-27,33$ \\
Usuario & R10 & 1,63 & 0,52 & 0,71 & $-68,10$ \\
\hline
\end{tabular}

Tabla 6. Tipología de los registros en porcentaje, según fase.

Las diferencias en la distribución según tipología de registros son notables entre la fase presencial y online. Acceder al sistema (R8) acumula el $40,75 \%$ y el $44,86 \%$ según la fase, con un aumento del $10,09 \%$, mientras que en los recursos docentes (R7) se ha accedido al $23,35 \%$ en la fase presencial y el $21,33 \%$ en la online, con una caída del $8,65 \%$, explicable porque muchos de los recursos ya estaban disponibles desde el comienzo del curso. En cuanto a las tareas (R9) han decaído el $27,33 \%$ sustituidas por un aumento del $29,31 \%$ en el uso de los cuestionarios (R4) que pasa del $9,54 \%$ en la fase presencial al $12,31 \%$ en la online. Igualmente, el uso del chat y el foro (R2) pasa del $8,82 \%$ al $6,10 \%$ con una caída del $30,84 \%$ para ser sustituido por el uso del Collaborate como 
herramienta docente online $(2,73 \%$ en fase presencial y $5,13 \%$ en online, con una tasa de variación del 84,53\%).

Estas diferencias en los usos de las diversas herramientas disponibles en el campus virtual, especialmente las que tienen que ver con la actividad docente (Chat, Collaborate) y las referidas a las actividades de evaluación (Tareas y Cuestionarios), muestran la temporalidad del proceso de evaluación de la asignatura, condicionada al cronograma de actividades amoldado a la situación real de la pandemia, sustituyendo pruebas de evaluación presenciales por las online, realizadas durante las dos últimas semanas lectivas de marzo y el mes de mayo de 2020 (tabla 7 y gráfico 6).

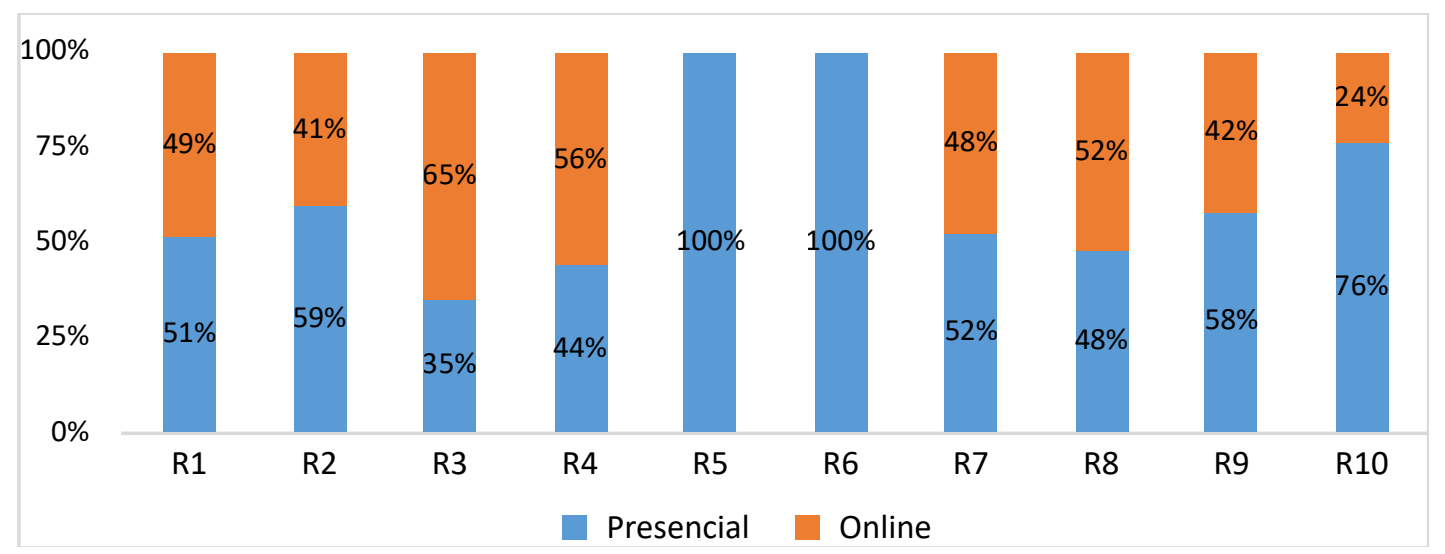

Gráfico 6. Distribución de los tipos de registros sobre el 100\%.

\begin{tabular}{lcccc}
\hline & \multicolumn{2}{c}{$\begin{array}{c}\text { Presencial } \\
\text { \%Total }\end{array}$} & \multicolumn{2}{c}{$\begin{array}{c}\text { Online } \\
\text { \%oTotal }\end{array}$} \\
\cline { 2 - 5 } Archivos enviados & ALUMNO & PROFESOR & ALUMNO & PROFESOR \\
\cline { 2 - 5 } Chat, Foro & 2,44 & 0,00 & 2,25 & 0,01 \\
Collaborate & 9,66 & 6,83 & 6,63 & 4,70 \\
Cuestionario & 3,01 & 2,22 & 6,95 & 0,21 \\
Informe general & 13,00 & 1,30 & 15,40 & 4,00 \\
Papelera de reciclaje & 0,02 & 0,11 & 0,00 & 0,00 \\
Recursos, glosario, Url & 0,00 & 0,740 & 0,00 & 0,00 \\
Sistema & 1,70 & 75,53 & 0,47 & 77,53 \\
Tarea, Detector plagio Unicheck & 52,04 & 14,09 & 56,52 & 13,42 \\
Usuario & 15,81 & 0,18 & 10,83 & 0,15 \\
\hline Total & 2,33 & 0,00 & 0,71 & 0,00 \\
\hline
\end{tabular}

Tabla 7. Tipología de registros del curso y distribución por profesor o alumno. 
Para el total de los usuarios, profesor y alumnos, (tabla 7) los accesos son mayoritariamente a los módulos del sistema, representando conjuntamente el $40,75 \%$ en la fase presencial y el $44,86 \%$ en la online, si bien el comportamiento difiere según el rol: así, para los alumnos representan el $52,04 \%$ en la fase presencial ( $56,52 \%$ en online) mientras que el profesor únicamente registra el $14,09 \%$ en la fase presencial $(13,42 \%$ en online), casi cuatro veces menos. En cuanto a los recursos docente, glosario y Url, el profesor accede el 75,53\% en la fase presencial, y ligeramente más, el $77,53 \%$ en la online, aunque los estudiantes registran únicamente el $1,70 \%$ en la fase presencial y menos aún en la fase online $(0,47 \%)$, lo cual es explicable dado que en las primeras semanas lectivas los estudiantes se descargan los recursos y es el profesor quien necesita ir ordenando, modificando, actualizando y completándolos.

En cuanto a las tareas de evaluación, los estudiantes acceden el $15,81 \%$ en la fase presencial, y menos intensamente en la fase online $(10,83 \%)$, siendo minoritaria el acceso del profesor $(0,18 \mathrm{y}$ $0,15 \%$ respectivamente). A los cuestionarios de evaluación los estudiantes acceden el $13,00 \%$ en fase presencial e intensifican al 15,40 en la online, mientras que el docente registra solo el 1,3\% en la fase presencial y se multiplica por 3 en la fase online $(4,00 \%)$. Por último, las herramientas de chat y foro, se utilizan más intensamente en la fase presencial, y computan el 9,66\% de los registros de los alumnos en la fase presencial frente al 6,63\% en la online, mientras que los docentes registran el $6,83 \%$ y $4,70 \%$ respectivamente en fase presencial y online. Del resto de tipología las diferencias entre ambos colectivos son destacables en el uso del Collaborate, que se duplica en la fase online para los estudiantes, pasando de 3,01 a 6,95\%, mientras que el docente reduce su participación relativa, pasando del $2,22 \%$ al $0,21 \%$, siendo el resto minoritarias.

Se evidencia un uso polarizado del campus virtual entre usuarios, no en los mismos tipos de registros, según la fase, presencial u online, y muestra el distinto trabajo que realizan en el entorno del campus virtual. Se confirma que el estudiante conectado al campus virtual exhibe un uso bastante concentrado de las plataformas condicionado al cronograma de actividades docentes y de evaluación, con un patrón de uso diferenciado respecto al profesor.

El reto de los docentes, ante esta pandemia covid-19 ha sido adaptar la docencia presencial a la online y principalmente abordar la evaluación online masiva, para lo cual se necesita la colaboración en un tándem perfecto del profesorado y sus estudiantes, dando respuestas al encaje de la metodología de enseñanza-aprendizaje y la tecnología, con garantías de equidad, seguridad jurídica y la transparencia (García-Peñalvo, Corell, Abella-García y Grande, 2020).

\section{Discusión final y conclusiones}

En los últimos meses se ha vivido una etapa excepcional por declaración de la pandemia por el COVID-19 que ha supuesto un reto a la implantación masiva de la transformación de la educación presencial a la online y la capacidad de reacción y ajuste, tanto de las instituciones educativas como del claustro docente, sin obviar el esfuerzo que han realizado estudiantes. Esta situación sobrevenida se ha acompañado de una acción de urgente reajuste, fuera de una ruta específicamente concebida, diseñada, valorada y planificada previamente para una docencia online (Hodges, Moore, Lockee, Trust y Bond, 2020). Las herramientas de docencia digital disponibles generan una ingente cantidad de información a partir de los registros de acceso y uso, permitiendo una extensa investigación en áreas diversas, desde la implementación de nuevas metodologías docentes, más interactivas, que requieren de una validación y contraste de la capacidad de aprendizaje autónomo, hasta el diseño de estrategias de análisis descriptivos (en su caso predictivos y de intervención) de los registros de los cursos con soporte online. 
En este contexto el docente se ha adaptado a la nueva realidad, para mitigar en lo posible el efecto de la pandemia en los estudiantes, intensificando el desempeño de nuevos roles centrados en 1) diseñar estrategias metodológicas y recursos online, sin esperar la validación técnica de la idoneidad de las mismos; 2) diseñar métodos de evaluación de conocimientos y competencias, llevados más por la propia intuición que por un modelo pedagógico de referencia, en correspondencia con Prensky (2013), para quien el docente además de ser experto en los contenidos de la materia que imparte, es también competente digital, experto instructor tanto en el uso de la tecnología educativa como en las metodologías activas, en aras a mejorar el proceso de enseñanza-aprendizaje (Bates, 2015). El resultado observado en estos meses está siendo muy clarificador de los pasos que todos, instituciones y claustros docentes deben seguir dando para avanzar en la digitalización de la educación en respuesta a las necesidades futuras, bajo el soporte de un modelo pedagógico de referencia.

El uso de las herramientas digitales registra información suficiente para observar diversos aspectos del proceso seguido por los estudiantes según los métodos de aprendizaje implantados, aportando visiones descriptivas y cuantitativas de su comportamiento, en algunos casos con carácter predictivo. Así, el LA sistematiza la información registrada en los entornos educativos virtuales pudiendo ser aplicada tanto para identificar brechas o diferenciales en los usos, como en cuantificar elementos del rendimiento de la educación (Lodge y Corrin, 2017). Los estudiantes muestran patrones diferenciados de interacción con los recursos docentes, en forma, ritmo y frecuencia, según su tipo de inteligencia y modo de aprendizaje, pudiendo estar vinculado al grado de desarrollado de diversas competencias incluidas las personales y sociales, más allá de las estrictamente digitales.

Este trabajo se inserta en esta línea de investigación del LA con un estudio a nivel micro o de caso, aporta evidencia sobre los cambios en los patrones del uso digital que tanto los estudiantes como los docentes realizan en la adaptación de la docencia presencial con apoyo en la herramienta Moodle a la online, consecuencia de la interrupción de las clases presenciales por el covid-19. El objetivo es conocer en profundidad aspectos del proceso de enseñanza-aprendizaje seguido y generar feedback formativo útil, que sirva de guía para diseñar futuros escenarios que requieran pasar de un entorno presencial a online, en línea con Harrison y Killion (2007) que describen al profesor como un observador de su propia acción docente, a partir de la investigación sobre los usos de sus estudiantes, tipología, resultados académicos, competencias adquiridas, e incluso compromisos y actitudes adoptadas con sus estudios y la institución educativa.

Las conclusiones del trabajo apuntan que el uso digital propiciado por el paso de la docencia presencial a la online provocado por el estado de alarma por COVID-19 muestra claras diferencias entre los usuarios de la plataforma Moodle, estudiantes y docente, tanto en frecuencia temporal como en la tipología de los recursos utilizados. Así, en la asignatura de referencia, las 8 semanas del periodo lectivo online multiplica casi por 5 los registros digitales, dada la presión que el proceso de ajuste ha supuesto sobre el campus virtual. Dicho uso difiere según el rol, dado que el docente llega a multiplicar sus registros por 10,63 veces, mientras que los estudiantes solo por 4,70 veces.

Los perfiles digitales muestran asimetrías según la fase presencial y online, mayor en la fase online: la mayor desigualdad se observa en el cuartil Q1 de la distribución, que se multiplica por 6,42 veces en la fase online respecto de la presencial (4,65 y 4,55 veces respectivamente en el Q2 y Q3), siendo los estudiantes del cuartil Q1 los que más reactivan su actividad digital. El índice de concentración CR10 se reduce del $27,07 \%$ en la fase de docencia presencial al $25,75 \%$ en la online, junto con un índice Herfindahl que determinan un número equivalente de alumnos respectivamente de 57,88 y 60,03 .

Los alumnos más y menos activos no son los mismos en las fases presencial y online, lo cual muestra un cambio de actitud y compromiso con el programa docente online seguido. La media sema- 
nal de registros para los 10 estudiantes más activos en la fase presencial es de 31,37 registros llegando a 140,35 en la fase online, y el cálculo del indicador de brecha digital (Iglesia, 2019) pasa de 138,22 en la fase presencial a 126,63 en la online, siendo los alumnos de las tipologías proactivos, sobreactivos y subactivos los que muestran una brecha digital más moderada en la fase online y al contrario entre los activos e inactivos.

Respecto a la naturaleza de los registros, los registros de las tareas decaen el $27,33 \%$, sustituido por el aumento del $29,31 \%$ del uso de cuestionarios en la fase online, el uso del chat y el foro también se reduce en el 30,84\% para ser sustituido por el uso del Collaborate como herramienta docente online con un incremento del $84,53 \%$. Este retrato muestra relevantes cambios en los usos de las herramientas disponibles en la plataforma del Moodle del campus virtual de la asignatura, que difieren además entre los diversos roles con los que se accede, condicionado al cronograma de las actividades docentes y de evaluación programadas.

Algunas de las conclusiones señaladas perfilan futuras investigaciones, en el contexto de Ferguson, Brasher, Clow y otros (2016), que aborden aspectos más concretos de los usos digitales de los estudiantes, referidos al rendimiento académico y evaluación del desarrollo de competencias, que requerirá disponer de información individual e institucional más completa que excede las limitaciones de éste estudio de caso. Disponer de estos estudios, periódicamente, en fases tempranas, sincronizado al avance del curso, aportará la información necesaria para diseñar acciones de intervención para adaptarse a circunstancias exógenas sobrevenidas, como ha sido la pandemia COVID-19, o detectar comportamientos erráticos o grados de subactividad de los estudiantes.

\section{Referencias}

Baker, R.S., Inventado, P.S. (2014). Educational data mining and learning analytics. In Learning Analytics; Springer: Berlin, Germany, pp. 61-75.

Banihashem S.K., Aliabadi K., Ardakani S. P., Ahmadabadi M.N. y Delavar A. (2019) Investigation on the Role of Learning Theory in Learning Analytics. Interdisciplinary Journal of Virtual Learn Medical Sciences. 10(3):1-14. DOI: $10.30476 /$ ijvIms.2019.84294.1001

Banihashem S.K., Aliabadi K., Ardakani S.P., Delaver A., Ahmadabadi M.N. (2018) Learning analytics: A critical literature review. Interdisciplinary Journal of Virtual Learning in Medical Sciences. 2018; 9(2). https:// dx.doi.org/10.5812/ijvIms.63024

Bates, A.W. (T) (2015). Teaching in a Digital Age: Guidelines for designing teaching and learning for a digital age. University of British Columbia. https://opentextbc.ca/teachinginadigitalage/

Berners-Lee, T., Hendler, J., y Lassila, O. (2001). The semantic web, Scientific American 284 (5): 28-37.

Buckingham S. y Ferguson, R. (2012). Social Learning Analytics. Educational Technology \& Society, 15 (3), 3-26. ISSN: 1436-4522. http://www.ifets.info/journals/15_3/2.pdf.

Campbell, J. P. (2007). Utilizing Student Data within the Course Management System to Determine Undergraduate Student Academic Success: An Exploratory Study, PhD, Purdue University.

Recuperado de https://docs.lib.purdue.edu/dissertations/AAI3287222/

Campbell, J. P., DeBlois, P. B. y Oblinger, D. G. (2007). Academic analytics: A new tool for a new era. EDUCAUSE Review, 42(4), 40.

Chick, R.C., Clifton, G.T., Peace, K.M., Propper, B.W., Hale D.F., Alseidi, A.A., y Vreeland, T.J. (2020) .Using Technology to Maintain the Education of Residents During the COVID-19 Pandemic, Journal of Surgical Education, Volume 77, Issue 4, pp.729-732, https://doi.org/10.1016/j.jsurg.2020.03.018 
Cocea, M. y Weibelzahl, S. (2007). Cross-system validation of engagement prediction from log files. Creating new learning experiences on a global scale. Springer Berlin Heidelberg, 14-25.

https://link.springer.com/chapter/10.1007/978-3-540-75195-3_2

Davis, K. (2012). Ethics of Big Data: Balancing Risk and Innovation. Ed. O'Reilly Media, Inc., USA, United States.

Drachsler, H. y Greller, W. (2016). Privacy and Analytics - it's a DELICATE issue. A Checklist to establish trusted Learning Analytics. 6th Learning Analytics and Knowledge Conference 2016, April 25-29, 2016, Edinburgh, UK.

Durall, E., y Gros, B. (2014). Learning analytics as a metacognitive tool. In Proceedings of 6th International Conference on Computer Supported Education Vol 1: CSEDU 380-384. https://www.scitepress.org/ Link.aspx?doi $=10.5220 / 0004933203800384$

Ferguson, R., Brasher A., Clow, D. y otros (2016). Research Evidence on the Use of Learning Analytics - Implications for Education Policy. Vuorikari y Castaño Muñoz (Eds.). Joint Research Centre Science for Policy Report; https://doi.org/10.2791/955210

Fernández Enguita, M. (2020). Una pandemia imprevisible ha traído la brecha previsible. Recuperado de https:// bit.ly/2VT3kzU

García-Peñalvo, F. J. (2020). El sistema universitario ante la COVID-19: Corto, medio y largo plazo. En: Universidad. Disponible en: https://bit.ly/2YPUeXU.

García-Peñalvo, F. J., Corell, A., Abella-García, V., y Grande, M. (2020). La evaluación online en la educación superior en tiempos de la COVID-19. Education in the Knowledge Society, 21, 12. https://doi.org/ $10.14201 /$ eks. 23086

Gašević D., Dawson S. y Rogers T. (2016). Learning analytics should not promote one size fits all: The effects of instructional conditions in predicting academic success. The Internet and Higher Education. Jan 1; 28:68-84. https://doi.org/10.1016/j.iheduc.2015.10.002

Gašević D., Dawson S., Siemens G.(2015). Let's not forget: Learning analytics are about learning. TechTrends. 2015 Jan 1;59(1):64-71. https://doi.org/10.1007/s11528-014-0822-x

Gros, B. (2016). The design of smart educational environments. Smart Learning Environments, 3(15), 1-11. https://doi.org/10.1186/s40561-016-0039-x

Harrison, C. y Killion, J. (2007). Ten roles for teacher leaders. Educational Leadership, 65(1) (September) 74-77.

http://www.ascd.org/publications/educational-leadership/sept07/vol65/num01/ten-roles-for-teacher-leaders.aspx

Herodotou, C.; Rienties, B.; Verdin, B.; Boroowa, A. (2019). Predictive learning analytics 'at scale': Towards guidelines to successful implementation in Higher Education based on the case of the Open University UK. Journal of learning Analytics, 6, pp. 85-95. https://doi.org/10.18608/jla.2019.61.5

Hodges, C.,Moore, S.,Lockee, B.,Trust, T. y Bond, A. (2020). The difference between emergency remote teaching and online learning. Educause Review. Recuperado de https://bit.ly/3b0Nzx7

Iglesia, M. C. (2019). Learning Analytics para una visión tipificada del aprendizaje de los estudiantes. Un estudio de caso. Revista Iberoamericana de Educación, 80(1), 55-87. https://doi.org/10.35362/rie8013444

Iglesia, M. C. (2020). Analítica de los usos digitales y rendimiento académico. Un estudio de caso con estudiantes universitarios. REIRE, Revista d'Innovació i Recerca en Educació, Vol. 13, Núm. 2 (2020). https:// doi.org/10.1344/reire2020.13.229267

Kay, D., Kom, N. and Oppenheim C. (2013). Legal, Risk and Ethical Aspects of Analytics in Higher Education. Analytics Series. Accessed January 3, 
https://web.archive.org/web/20130502234313/http://publications.cetis.ac.uk/wp-content/uploads/ 2012/11/Legal-Risk-and-Ethical-Aspects-of-Analytics-in-Higher-Education-Vol1-No6.pdf

Knight S., Shum S.B., Littleton K. (2014). Epistemology, assessment, pedagogy:where learning meets analytics in the middle space. Journal of Learning Analytics. 2014 Aug 7;1(2): 23-47. https://doi.org/10.18608/ jla.2014.12.3

Li, Q.; Baker, R.; Warschauer, M. (2020). Using clickstream data to measure, understand, and support self-regulated learning in online courses. Internet Hight Education, 100727.

Liz-Domínguez, M., Caeiro-Rodríguez, M., Llamas-Nistal, M., Mikic-Fonte, F.A. (2019). Systematic Literature Review of Predictive Analysis Tools in Higher Education. Applied. Science. 9, 5569.

Lodge, J. M., y Corrin, L. (2017). What data and analytics can and do say about effective learning. Npj Science of Learning, 2(1). https://doi.org/10.1038/s41539-017-0006-5

Long, Ph. y Siemens, G. (2011). Penetrating the Fog: Analytics in Learning and Education. EDUCAUSE Review, 46, 5, 30-40. https://er.educause.edu/articles/2011/9/penetrating-the-fog-analytics-in-learning-andeducation

Mazza, R. y Botturi, L. (2007). Monitoring an Online Course with the GISMO Tool: A Case Study. Journal of Interactive Learning Research (2007), 18 (2), 251-265.

Mazza R. y Milani, C. (2004). GISMO: a Graphical Interactive Student Monitoring Tool for Course Management Systems. T.E.L.'04 Technology Enhanced Learning '04 International Conference. Milan, 18-19 November 2004.

OECD (2019). Benchmarking Higher Education System Performance; Paris, France, 2019; p. 644, https:// doi.org/10.1787/be5514d7-en.

Pardo, A. (2018). A feedback model for data-rich learning experiences. Assessment \& Evaluation in Higher Education, 43(3), 428-438. https://doi.org/10.1080/02602938.2017.1356905

Pardo, A. y Siemens, G. (2014). Ethical and privacy principles for learning analytics. British Journal of Educational Technology, 45(3), 438-450. https://doi.org/10.1111/bjet.12152

Picciano, A. (2012). The Evolution of Big Data and Learning Analytics in American Higher Education. Journal of Asynchronous Learning Networks, 16(3), 9-20.

Picciano, A. G. (2014) Big Data and Learning Analytics in Blended Learning Environments: Benefits and Concerns. International Journal of Artificial Intelligence and Interactive Multimedia, Vol. 2, No 7, pp 35-43. http://doi.org/10.9781/ijimai.2014.275

Prensky, M. (2013). Enseñar a nativos digitales (1a. ed). México: SM Ediciones.

Ramos, C. y Yudko, E. (2008). "Hits" (not "Discussion Posts") predict student success in online courses: A doublé cross-validation study. Computers \& Education, 50(4):1174-1182.

https://doi.org/10.1016/j.compedu.2006.11.003

Romero, C., Ventura, S. y Garcia, E. (2008). Data mining in course management systems: Moodle case study and tutorial. Computers \& Education, 51(1), 368-384. https://doi.org/10.1016/j.compedu.2007.05.016

Sanger, D.E. y Perlroth, N. (2014). Internet Giants Erect Barriers to Spy Agencies. New York Times. (June 6, 2014)

https://www.nytimes.com/2014/06/07/technology/internet-giants-erect-barriers-to-spy-agencies.html

Sampson, D. (2017). Teaching and learning analytics to support teacher inquiry. In 2017 IEEE Global Engineering Education Conference (EDUCON 2017), Apr 25, 2017, Athens, Greece.

Siemens G. (2013). Learning Analytics: The Emergence of a Discipline, American Behavioral Scientist. Vol 57(10), p.1380-1400, SAGE Publications.

http://journals.sagepub.com/doi/abs/10.1177/0002764213498851?journalCode=absb 
Slade, S. y Prinsloo, P. (2013). Learning Analytics: Ethical Issues and Dilemmas. American Behavioral Scientist, 57 (10), 1510-1529. https://doi.org/10.1177\%2F0002764213479366

Stewart, C. (2017). Learning Analytics: Shifting from theory to practice. Journal on Empowering Teaching Excellence, 1(1), 95-105. https://doi.org/10.15142/T3G63W

Tempelaar, D. (2020) Supporting the less-adaptive student: the role of learning analytics, formative assessment and blended learning, Assessment \& Evaluation in Higher Education, 45:4, 579-593, https://doi.org/ 10.1080/02602938.2019.1677855

Xing, W., Guo, R., Petakovic, E., y Goggins, S. (2015). Participation-based student final performance prediction model through interpretable Genetic Programming: Integrating learning analytics, educational data mining and theory. Computers in Human Behavior, 47, 168-181. https://doi.org/10.1016/ j.chb.2014.09.034

Yukselturk, E. Ozekes, S. y Turel, Y. K. (2014) Predicting Dropout Student: An Application of Data Mining Methods in an Online Education Program. European Journal of Open, Distance and E-Learning, 17, 1, pp. 118-133. https://doi.org/10.2478/eurodl-2014-0008 